\title{
Glutathione deficiency in type 2 diabetes impairs cytokine responses and control of intracellular bacteria
}

\author{
Kai Soo Tan, ${ }^{1}$ Kok Onn Lee, ${ }^{2}$ Kee Chung Low, ${ }^{1}$ Akshamal Mihiranga Gamage, ${ }^{1}$ Yichun Liu, ${ }^{3}$ \\ Gek-Yen Gladys Tan, ${ }^{3}$ Hui Qi Vanessa Koh,4,5 Sylvie Alonso, ${ }^{4,5}$ and Yunn-Hwen Gan 1,5 \\ ${ }^{1}$ Department of Biochemistry and ${ }^{2}$ Department of Medicine, Yong Loo Lin School of Medicine, National University of Singapore, Singapore. \\ ${ }^{3}$ Defence Medical and Environmental Research Institute, Defence Science Organization National Laboratories, Singapore. ${ }^{4}$ Department of Microbiology, \\ Yong Loo Lin School of Medicine, and 5 Immunology Program, National University of Singapore, Singapore.
}

\begin{abstract}
Individuals with type 2 diabetes are at increased risk of acquiring melioidosis, a disease caused by Burkbolderia pseudomallei infection. Although up to half of melioidosis patients have underlying diabetes, the mechanisms involved in this increased susceptibility are unknown. We found that B. psendomallei-infected PBMCs from diabetic patients were impaired in IL-12p70 production, which resulted in decreased IFN- $\gamma$ induction and poor bacterial killing. The defect was specific to the IL-12-IFN- $\gamma$ axis. Defective IL-12 production was also observed during Mycobacterium tuberculosis infection, in which diabetes is likewise known to be a strong risk factor. In contrast, IL-12 production in diabetic cells was not affected upon Salmonella enterica infection or in response to TLR2, -3, -4 , and -5 ligands. Poor IL-12 production correlated with a deficiency in intracellular reduced glutathione (GSH) concentrations in diabetic patients. Addition of GSH or N-acetylcysteine to PBMCs selectively restored IL-12 and IFN- $\gamma$ production and improved bacterial killing. Furthermore, the depletion of GSH in mice led to increased susceptibility to melioidosis, reduced production of IL-12p70, and poorer disease outcome. Our data thus establish a link between GSH deficiency in diabetes and increased susceptibility to melioidosis that may open up new therapeutic avenues to protect diabetic patients against some intracellular bacterial pathogens.
\end{abstract}

\section{Introduction}

Type 2 diabetes mellitus has been described as a global epidemic fueled by population growth, aging, urbanization, and increasing obesity. It is estimated that the number of people diagnosed with diabetes is expected to grow from 171 million in 2000 to $366-440$ million by 2030, with three-quarters of the patients living in lowincome countries (1). Besides being associated with major complications, such as cardiovascular disease, atherosclerosis, retinopathy, nephropathy, and neuropathy (2-5), prevalence of infections is also much more common in diabetic individuals. This increased susceptibility to infection has been attributed to neutrophil dysfunction, dehydration, malnutrition, vascular insufficiency, and neuropathy (6). One of the infectious diseases in which type 2 diabetes is identified as the strongest risk factor is melioidosis, caused by the Gram-negative bacterium Burkholderia psendomallei. Melioidosis is endemic in southeast Asia, northern Australia, southern China, and India, but has also been increasingly reported in Africa, the Middle East, and South America (7-9). In hyperendemic regions, such as northeastern Thailand, up to $60 \%$ of melioidosis patients have underlying type 2 diabetes (8). In the state of Kedah in Malaysia, $57 \%$ of all diagnosed cases had underlying type 2 diabetes (10). The disease is difficult to treat due to the inherent antibiotic resistance of the bacterium, and treatment regimens involve prolonged antibiotic eradication therapies lasting months (8). With increasing incidence of diabetes in many of these lower income countries (1), the coincidence of diabetes with melioidosis looks set to increase and could present a financial and health care burden to resource-strapped regions.

Conflict of interest: The authors have declared that no conflict of interest exists. Citation for this article: J Clin Invest. 2012;122(6):2289-2300. doi:10.1172/JCI57817.
B. pseudomallei is a facultative intracellular microbe with broad host and tissue tropism. It causes a wide spectrum of disease manifestations ranging from asymptomatic seroconversion, acute or chronic pneumonia, localized infection involving one organ, and disseminated septicemic disease involving multiple organs to septic shock (7). Relapse of disease could occur many years after primary infection (7). The mortality rate remains as high as $40 \%$ in areas of hyperendemicity for septicemic disease (8). The strong association between type 2 diabetes and melioidosis presents a unique opportunity to identify, in diabetes, susceptibility factors to infection and disease. No studies have explored the mechanism for increased susceptibility to melioidosis in diabetic patients except for a recent report that found neutrophils isolated from diabetic individuals to be defective in chemotaxis, phagocytosis, and apoptosis toward B. pseudomallei (11).

Type 2 diabetes is increasingly being recognized as a disorder of the innate immune system characterized by a chronic low level of inflammation and deregulation of the inflammasome (12). Besides neutrophils, other cell types, such as macrophages and cytokines, such as IFN- $\gamma$, have been shown to be important for the innate host defense against $B$. psendomallei $(13,14)$. To discover host susceptibility factors that would predispose diabetic individuals to $B$. psendomallei infections, we screened the immune profile of PBMCs isolated from diabetic patients and their ageand sex-matched healthy controls in response to B. pseudomallei. We found that defective IL-12 and therefore IFN- $\gamma$ production by cells from diabetic patients led to poor control of intracellular bacterial replication. Furthermore, we were able to trace the defective IL-12 response to a deficiency in intracellular reduced glutathione (GSH) in diabetic cells not only in response to B. pseudomallei but also to Mycobacterium tuberculosis. 


\section{Table 1}

Characteristics of the study population

\begin{tabular}{lccc} 
Group & Age & Male/female & HbA1c \\
Healthy control $(n=45)$ & $48.8 \pm 7.7$ & $28 / 17$ & ND \\
Poor control $(n=45)$ & $50.3 \pm 7.7$ & $28 / 17$ & $9.99 \pm 1.99$ \\
Good control $(n=9)$ & $49.1 \pm 6.8$ & $4 / 5$ & $6.64 \pm 0.46$ \\
\hline
\end{tabular}

Values are expressed as mean \pm SD. ND, not determined.

\section{Results}

Poor intracellular bacterial control is due to impaired IL-12 and IFN- $\gamma$ production in PBMCs from diabetic individuals. To determine the mechanisms underlying the strong association of melioidosis with type 2 diabetes, we investigated whether PBMCs isolated from diabetic and age- and sex-matched nondiabetic healthy volunteers (Table 1) show any differential capacity at controlling bacterial infection. PBMCs from the respective groups were infected with $B$. psendomallei, and the numbers of intracellular bacteria were enumerated 8 hours later. PBMCs from diabetic patients were found to harbor significantly more intracellular bacteria than their healthy counterparts (Figure 1A). This observation correlated with the poorer respiratory burst generated by the diabetic PBMCs in response to B. pseudomallei compared with healthy PBMCs, even though the baseline ROS production was higher in diabetic cells (Supplemental Figure 1; supplemental material available online with this article; doi:10.1172/JCI57817DS1). Since early control of bacterial infection involves the production of inflammatory cytokines, we screened for cytokine differences in PBMCs infected with B. psendomallei from 5 matched pairs of diabetic versus healthy individuals in order to identify the contributing factors to poor intracellular bacterial control in diabetic PBMCs. We found significantly lower IL-12 and IFN- $\gamma$ production in diabetic PBMCs, whereas other cytokines we profiled showed similarity (Supplemental Figure 2, A and B) between diabetic and healthy cells. The lower production of IL-12 and IFN- $\gamma$ in diabetic PBMCs upon B. pseudomallei infection was further confirmed in a bigger cohort of diabetic and healthy subjects (Figure 1, B and C).

Since IL-12 is a major inducer of IFN- $\gamma$ production, we determined whether the low IFN- $\gamma$ production in B. pseudomallei-infected PBMCs was a result of low IL-12 concentration. Neutralizing antibodies against $\mathrm{p} 40$ reduced $B$. pseudomallei-induced IFN- $\gamma$ production by $80 \%$ (Figure 1D). Although the neutralization could potentially affect IL-23 due to the shared p40 subunit (15), IL-23 was undetectable following B. psendomallei infection of PBMCs, making this possibility remote. Primed monocytes are known to be one of the primary sources of IL-12, and monocytes isolated from peripheral blood of diabetic patients showed a significantly lower production of IL-12 upon bacterial infection compared with those from healthy individuals, similar to what was observed with PBMCs (Figure 1E). In addition, quantitative real-time PCR analysis revealed that the transcript levels for the IL-12p35 subunit (IL12A) were significantly decreased in diabetic monocytes compared with healthy individuals, whereas the p40 (IL12B) transcript levels remained comparable (Figure 1, F and G).

Since IFN- $\gamma$ primes phagocytes for increased oxidative burst, the low production of IL-12 and IFN- $\gamma$ could be the cause of poor bacterial control in the diabetic cells. To investigate the correlation between poor control of bacterial replication and low levels

of IL-12 in diabetic PBMCs, IL-12 was added exogenously to the cells and the bacterial killing efficacy monitored. The number of intracellular bacteria was significantly reduced in both the healthy and diabetic groups compared with their respective controls (without exogenous IL-12) (Figure 2, A and B). The addition of exogenous IFN- $\gamma$ also significantly improved bacterial killing in both healthy and diabetic cells (Figure 2, C and D). Although the absolute number of bacteria was still higher in the diabetic cells, the diabetic cells could respond effectively to IL-12 and IFN- $\gamma$, and there were no inherent defects in these receptors or their signaling pathways. Together, these data demonstrate that PBMCs (and monocytes in particular) from diabetic subjects were impaired in the early control of $B$. psendomallei infection due to a lower production of IL-12, which led to subsequent poor IFN- $\gamma$ production. Furthermore, we showed that impaired receptor function or downstream signaling could not account for the production defect in both cytokines.

Selective defect in IL-12 production to B. pseudomallei and M. tuberculosis infection is MyD88 and TRIF (TIR domain-containing adaptor-inducing IFN $\beta$ ) independent. The poor IL-12 production in diabetic individuals could be a general defect or only in response to specific stimuli. To distinguish between these 2 possibilities, we compared infection of PBMCs from diabetic or healthy donors with B. psendomallei and 2 other intracellular pathogens, Salmonella enterica and M. tuberculosis. Defective IL-12 and IFN- $\gamma$ production was observed in cells infected with either B. psendomallei or M. tuberculosis, but not with S. enterica (Figure 3, A and B). Furthermore, IL-12 production in response to TLR2, -4 , and -5 agonists was comparable between diabetic and healthy monocytes. Together, these findings indicated that the lower IL-12 production observed with the diabetic cells is pathogen dependent and suggest that IL-12 production in response to $B$. pseudomallei and $M$. tuberculosis is TLR2, -4 , and -5 pathway independent (Figure 3C).

To determine whether the IL-12 response is dependent on the MyD88 signaling pathway, monocytes infected with B. pseudomallei or S. enterica were treated with a MyD88 peptide inhibitor. IL-12 production from $S$. enterica-infected cells was maximally inhibited by the peptide inhibitor to the same level as that of the cells stimulated with control TLR2 agonist, whereas IL-12 response from $B$. pseudomallei-infected cells was inhibited by about $60 \%$ (Figure 3D). Furthermore, the IL-12 response in B. pseudomallei-infected cells was inhibited by $50 \%$ with the TRIF peptide inhibitor and $100 \%$ with both MyD88 and TRIF inhibitors (Figure 3E). Thus, these data indicated that, whereas S. enterica-induced IL-12 production is fully MyD88-dependent, B. psendomallei-induced IL-12 relies on both MyD88 and TRIF signaling pathways. Furthermore, similar levels of IL-12 were measured with monocytes from diabetic and healthy donors stimulated with TLR3 ligand poly(I:C), which signals exclusively through TRIF (Figure 3F). This latest observation thus ruled out the possibility that a defect in the TRIF pathway would account for the impaired IL-12 production of diabetic cells in response to $B$. pseudomallei and $M$. tuberculosis. In conclusion, our data support that the impaired production of IL-12 in diabetic monocytes is pathogen specific and is not due to a defect in the MyD88 and TRIF signaling pathways.

Intracellular GSH levels selectively affect IL-12 from bacteria-infected cells. The selective defect in IL-12 induction in response to B. pseudomallei but not other MyD88-dependent stimuli suggests alterations in specific signaling pathways in diabetic cells. It is possible 

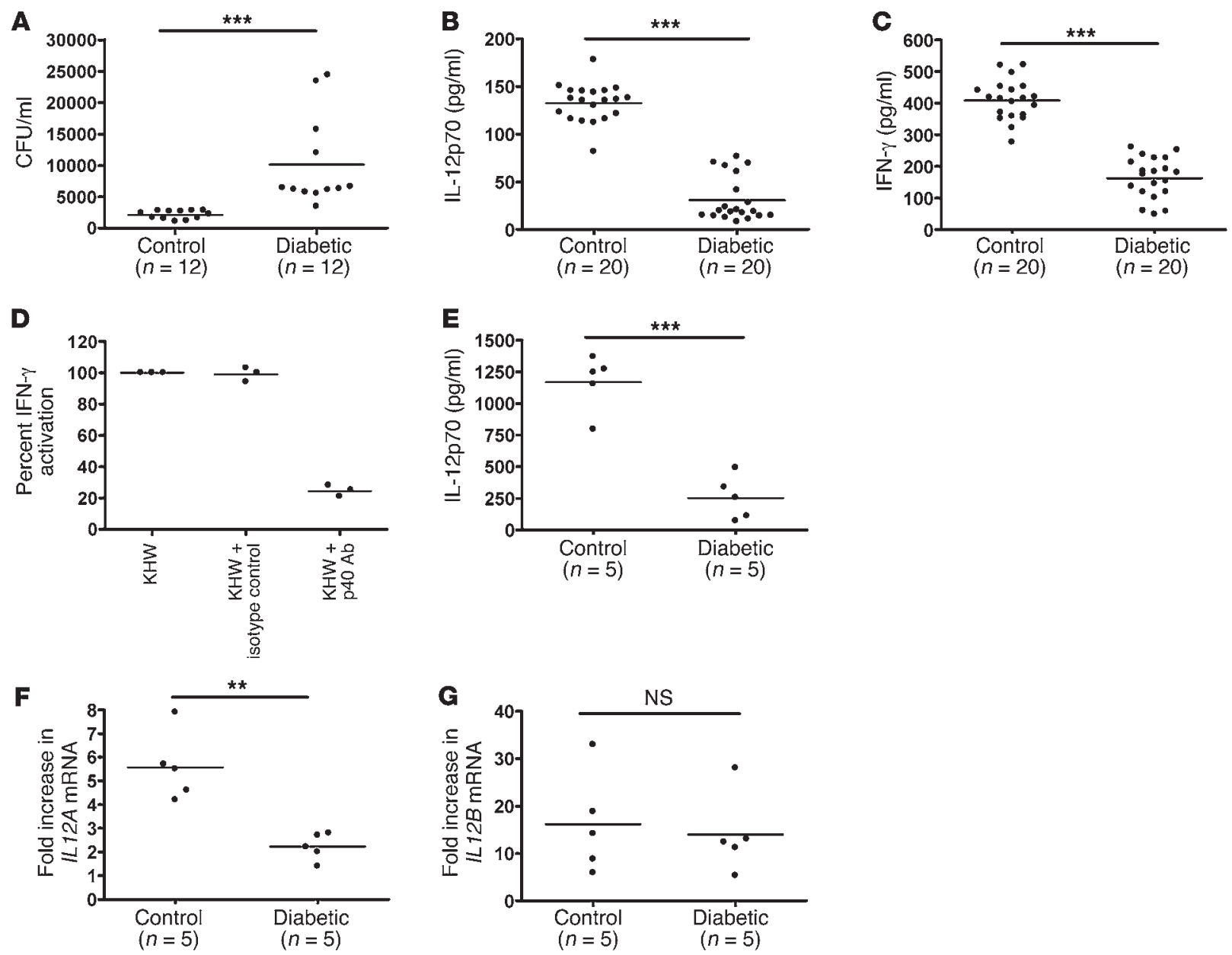

\section{Figure 1}

Decreased IL-12 and IFN- $\gamma$ production contribute to high intracellular bacterial loads in diabetic PBMCs. (A) PBMCs isolated from healthy or diabetic subjects were infected with $\mathrm{KHW}$, a strain of $B$. pseudomallei, at an $\mathrm{MOI}$ of 50:1 for 8 hours, and the amount of intracellular bacteria was enumerated by plating on TSA plates. (B) IL-12 and (C) IFN- $\gamma$ concentrations were determined by ELISA. (D) PBMCs from healthy donors $(n=3)$ were left untreated or pretreated for 4 hours with either isotype control $(10 \mu \mathrm{g} / \mathrm{ml})$ or p40 neutralizing antibody $(10 \mu \mathrm{g} / \mathrm{ml})$ prior to infection with B. pseudomallei. IFN- $\gamma$ concentrations were determined by ELISA 24 hours after B. pseudomallei infection. (E) Monocytes isolated from healthy or diabetic individuals were pretreated with recombinant IFN- $\gamma(100 \mathrm{ng} / \mathrm{ml}) 2$ hours prior to bacterial infection, and IL-12 concentrations were determined 24 hours after infection. Monocytes isolated from healthy or diabetic individuals were infected with $B$. pseudomallei for 6 hours after which (F) IL12A and (G) IL12B transcripts were determined by quantitative PCR. Each symbol represents data obtained from 1 study subject, and the horizontal lines denote the means for the respective experimental groups. ${ }^{* \star *} P<0.001 ;{ }^{* \star} P<0.01 ;$ n.s. $=P>0.05$.

that the changes wrought by hyperglycemia, such as increased oxidative stress or glycation of proteins in diabetes, could lead to signaling modulations affecting IL-12 (16). It was previously reported that LPS-induced IL-12 production from macrophages was selectively modulated by intracellular GSH concentrations (17). Since diabetic cells showed an increased oxidative stress with constitutive higher basal production of ROS (Supplemental Figure 1A), we reasoned that a GSH deficiency in diabetic cells may affect IL12 production in response to $B$. psendomallei infection. To test this hypothesis, $B$. psendomallei-infected PBMCs from healthy donors were incubated with cell-permeable reduced GSH or depleted of intracellular GSH via diethyl maleate (DEM) treatment. The addition of GSH significantly boosted the production of both IL-12 and IFN- $\gamma$ in B. pseudomallei-infected cells (Figure 4, A and B). Conversely, the addition of DEM reduced bacteria-induced IL-12 and
IFN- $\gamma$ (Figure 4, A and B). GSH addition had no effect on the other 10 cytokines profiled by multiplex ELISA (Figure 4C). Treatment with $\mathrm{N}$-acetylcysteine (NAC) also increased IL-12 and IFN- $\gamma$, providing further support that alteration of the cellular redox balance modulated the IL-12-IFN- $\gamma$ axis (Figure 4, D and E). A titration of both GSH and NAC concentrations indicated that $5 \mathrm{mM}$ is optimal, and increasing the concentrations led to a drop in IL-12 production (Figure 4F), in agreement with what had been previously reported (18).

The above observations prompted us to determine the intracellular levels of GSH in PBMCs isolated from diabetic and healthy donors. Diabetic patients were further divided into good and poor glycemic control groups based on their HbA1c levels, and all 3 groups of volunteers were matched according to sex and age. The ratios of GSH to oxidized GSH (GSSG) in cells from the 
A
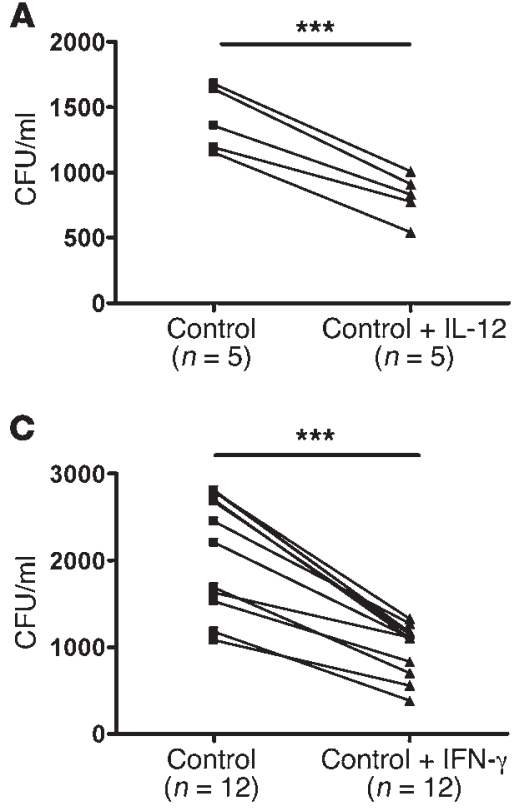

B
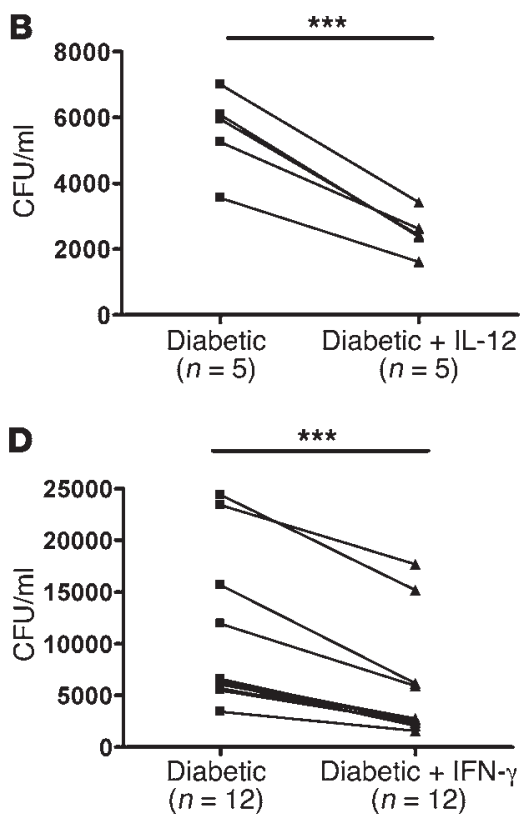

Figure 2

Defective IL-12 and IFN- $\gamma$ production contribute to high intracellular bacterial loads in diabetic PBMCs. Intracellular bacterial loads were determined as described above. PBMCs from either the (A and C) healthy or (B and $\mathbf{D}$ ) diabetic individuals were pretreated with recombinant (A and B) IL-12 (100 $\mathrm{ng} / \mathrm{ml})$ or $(\mathbf{C}$ and $\mathbf{D})$ IFN- $\gamma(100 \mathrm{ng} / \mathrm{ml}) 2$ hours prior to infection with $B$. pseudomallei, and the number of intracellular bacteria were determined as described above. Each symbol represents data from 1 study subject. Values obtained from the same study subject before and after cytokine treatment are joined by a line. ${ }^{* \star \star} P<0.001$. poor glycemic control group were significantly lower compared with those in the good glycemic control or the healthy groups, and the ratios of the good glycemic control subjects resembled those of the healthy donors (Table 2). Thus, a high GSH/GSSG ratio correlates with a lower $\mathrm{HbA} 1 \mathrm{c}$ level in diabetics. Although the good glycemic control subjects had the highest BMIs, which were significantly different from those of the healthy as well as the poor glycemic control subjects, their GSH/GSSG ratios were similar to those of the healthy subjects. This excluded obesity as a contributing factor.

Increasing GSH concentration in diabetic cells reverses low IL-12 production and improves intracellular bacterial control. In further examining the correlations in the 3 groups of donors, we found that the intracellular bacterial numbers in PBMCs of diabetic individuals with good glycemic control were significantly lower compared with those of individuals with poor glycemic control and were similar to those of the healthy group (Table 2). Consistently, the good glycemic control group and the healthy group showed significantly higher IL-12 and IFN- $\gamma$ production compared with the poor glycemic control group (Table 2). Therefore, low GSH/GSSG ratio in cells from diabetic subjects correlates with poor IL-12 and IFN- $\gamma$ production in response to $B$. pseudomallei and defective bacterial control. To ascertain whether low GSH concentration is responsible for poor IL-12 and bacterial control in the poor glycemic control group, cells from this group of diabetic donors were supplemented with GSH prior to infection with B. psendomallei. Diabetic cells treated with GSH showed significant improvement in the control of intracellular B. psendomallei, as evidenced by a drop in bacterial numbers (Figure 5A), establishing the causal effect of GSH on IL-12 and intracellular bacterial control in diabetes. Furthermore, while exogenous addition of GSH increased the levels of IL-12 and IFN- $\gamma$ in B. pseudomallei- and M. tuberculosis-infected cells, no effect was observed with $S$. enterica-infected cells (Figure 5, $\mathrm{B}$ and $\mathrm{C}$ ), thus confirming the existence of pathogen-specific signaling pathways of IL-12 production and variability among these pathways in their susceptibility to modulation by GSH. Furthermore, exogenous addition of GSH did not affect IL-12 production by poly(I:C)-treated PBMCs (Supplemental Figure 3), demonstrating that GSH does not directly modulate the TRIF pathway per se and confirming that the TRIF pathway is not involved.

GSH depletion results in increased susceptibility to melioidosis in mice. To establish a direct causal link between GSH deficiency and susceptibility to $B$. psendomallei infection in vivo, we chose to induce a state of GSH deficiency in a murine model of melioidosis rather than employing a diabetic mouse model because BM-derived DCs and peritoneal-elicited macrophages from diabetic mice induced by streptozotocin for 70 days showed significantly lower cytokine gene expression in many genes, such as TNFA, IL12B, IL1B, and IL18 in response to $B$. pseudomallei infection in the diabetic animals compared with healthy animals (19). In the BKS.Cg-Dock $7^{m}+/+$ Lepr $r^{d b} / \mathrm{J}$ mice, which carry a mutation in the leptin receptor, the homozygous $(d b / d b)$ mice were obese and showed increased TNFA, IL1B, and IL6 expression in response to $B$. psendomallei infection when compared with the heterozygous littermates even at time points when bacterial loads were comparable between the 2 groups (20). The broad differences seen in these models are different from what we found in our human study. The depletion of GSH was achieved by treating the mice with DEM/buthionine sulfoximine (BSO) before infection, as previously described $(21,22)$. Control mice treated with DEM/BSO, but not infected, remained well throughout the experiment. However, infected mice pretreated with DEM/BSO exhibited lethality at twice the rate of those without treatment, thus demonstrating that GSH deficiency results in increased susceptibility to disease (Figure 6A). At both time points after infection, a significantly higher bacterial load in the spleens of GSH-depleted animals was seen, especially at the earlier time point (Figure 6B). IL-12p70 was significantly decreased in the GSH-depleted animals at day 2 after infection (Figure 6C), and there was an obvious decrease in IFN- $\gamma$ production in most of these animals at day 2, although overall it did not approach statistical significance (Figure 6D). IFN- $\gamma$, IL-1 $\beta$, (Figure $6 \mathrm{E}$ ), and TNF- $\alpha$ (Figure $6 \mathrm{~F}$ ) were all increased significantly at day 1 after infection in the GSH-depleted animals, corresponding with the very significant increase in bacterial loads. It is well established that acute melioidosis induces hyperinflammation characterized by 
A
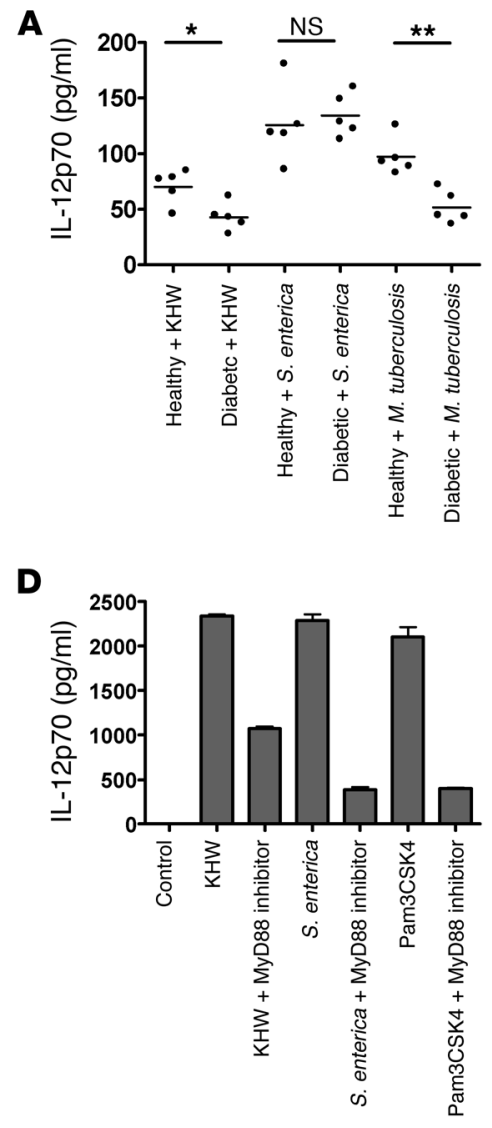
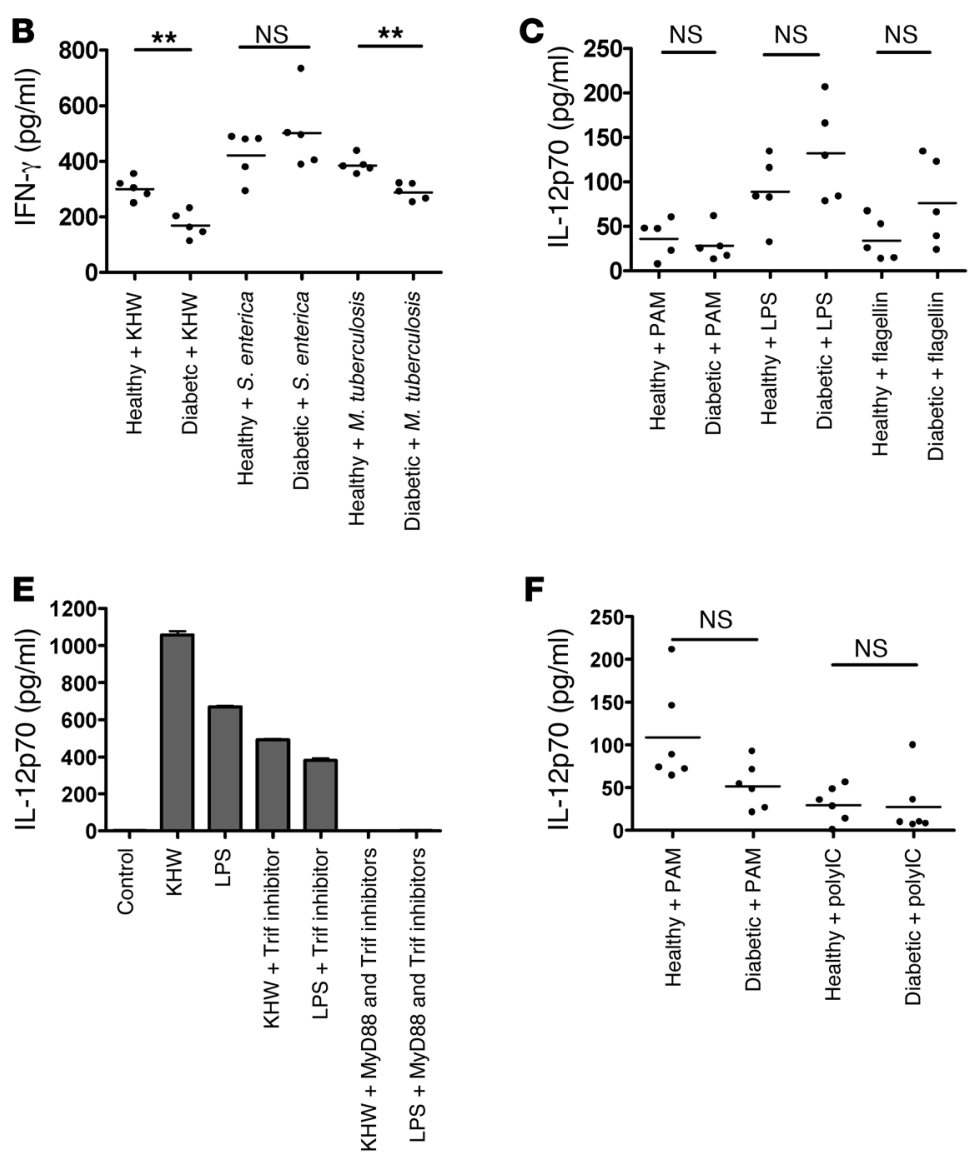

$\mathbf{F}$

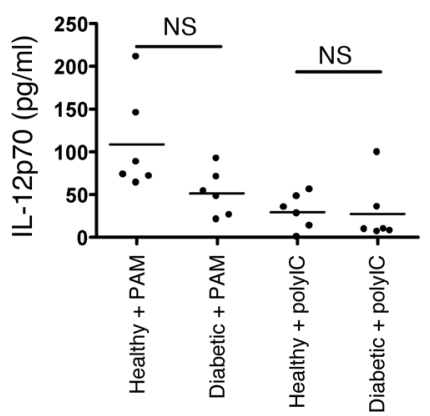

\section{Figure 3}

Diabetic PBMCs infected with B. pseudomallei or M. tuberculosis show defective IL-12 and IFN- $\gamma$ production. PBMCs isolated from healthy $(n=5)$ or diabetic $(n=5)$ individuals were infected ex vivo with either B. pseudomallei, S. enterica, or M. tuberculosis for 24 hours. (A) IL-12 and (B) IFN- $\gamma$ production were determined by ELISA. (C) Primary monocytes isolated from either healthy $(n=5)$ or diabetic $(n=5)$ individuals were treated with $\mathrm{PAM}_{3} \mathrm{CSK}_{4}(1 \mu \mathrm{g} / \mathrm{ml})$, UP-LPS $(1 \mu \mathrm{g} / \mathrm{ml})$, or flagellin $(1 \mu \mathrm{g} / \mathrm{ml})$ for 24 hours. (D) Primary monocytes isolated from a healthy donor were left untreated or treated with MyD88 inhibitor peptide $(100 \mu \mathrm{M}) 6$ hours prior to treatments with B. pseudomallei, S. enterica, or PAM $\mathrm{CSSK}_{4}$ for 24 hours. (E) Primary monocytes from a healthy donor were left untreated or pretreated with TRIF inhibitor peptide alone (25 $\mu$ M) or a mixture of TRIF $(25 \mu \mathrm{M})$ and MyD88 inhibitor $(100 \mu \mathrm{M})$ peptides 6 hours prior to KHW or LPS treatments. (F) Primary monocytes isolated from donors were stimulated with $\mathrm{PAM}_{3} \mathrm{CSK}_{4}(1 \mu \mathrm{g} / \mathrm{ml})$ or poly $(\mathrm{l}: \mathrm{C})(50 \mu \mathrm{g} / \mathrm{ml})$ for 24 hours. All experiments with primary monocytes involved priming the cells with IFN- $\gamma(100 \mathrm{ng} / \mathrm{ml})$. Each symbol represents data obtained from 1 study subject. Horizontal lines for the dot plots show the mean values for that particular experimental group. Data for the bar charts are presented as mean \pm SEM. ${ }^{* \star} P<0.01 ;{ }^{*} P<0.05 ;$ n.s. $=P>0.05$.

high levels of IFN- $\gamma$ in humans (23) and mice (24). In the infection model involving BALB/c and C57BL/6 mice, although the susceptible BALB/c mice produced less IL-12 than the relatively resistant C57BL/6 mice at all time points examined, they succumbed to high bacterial loads that corresponded with an early transient increase in IFN- $\gamma$ that was not seen in C57BL/6 mice (24). Similarly, it is likely that the hyperinflammation and transient surge in IFN- $\gamma$ caused by bacterial sepsis at day 1 masked the effects induced by GSH in the DEM/BSO-treated animals. At day 2 after infection, the effect of GSH became evident with the decrease in IL-12 and IFN- $\gamma$ in DEM/ BSO-treated animals. Consistent with our previous in vitro data, production of IL- $1 \beta$ and TNF- $\alpha$ was not affected by GSH depletion in the mice at day 2 after infection (Figure 6, E and F).

To separate the effect of bacterial-induced hyperinflammation and hyper-production of IFN- $\gamma$ from the primary effect of GSH depletion, we examined GSH depletion in splenocytes isolated from naive mice and infected in vitro with $B$. psendomallei.
We found that GSH depletion with $50 \mu \mathrm{M}$ of DEM consistently depleted the production of IFN- $\gamma$, but did not affect TNF- $\alpha$ concentration (Figure 6, G and H). Concentration of IL-12p70 was below the limit of detection by the ELISA kit. As treatment with DEM did not result in changes in the distribution of various cell types in the spleen (Supplemental Table 1), this shows that the decrease in IL-12 is not due to selective depletion of cell types. This is consistent with our claim that GSH depletion selectively reduces IL-12 and IFN- $\gamma$ production in response to B. pseudomallei infection.

\section{Discussion}

In this study, we have shown that low intracellular GSH concentrations in PBMCs from diabetic patients with poor glycemic control (HbA1c $\geq 8$ ) contribute to a significant decrease in IL-12 production, with reduced IL-12p35 transcription in response to B. psendomallei infection. The consequence of low IL-12 is a reduc- 

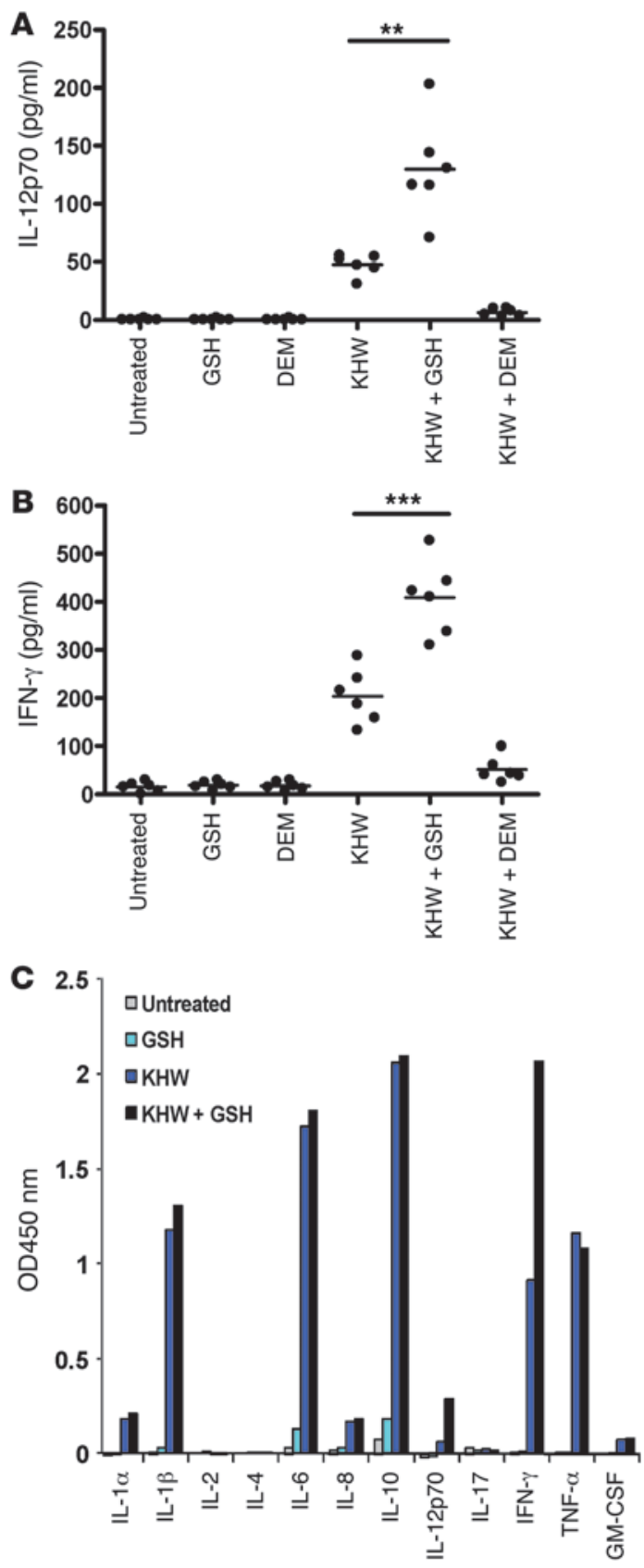
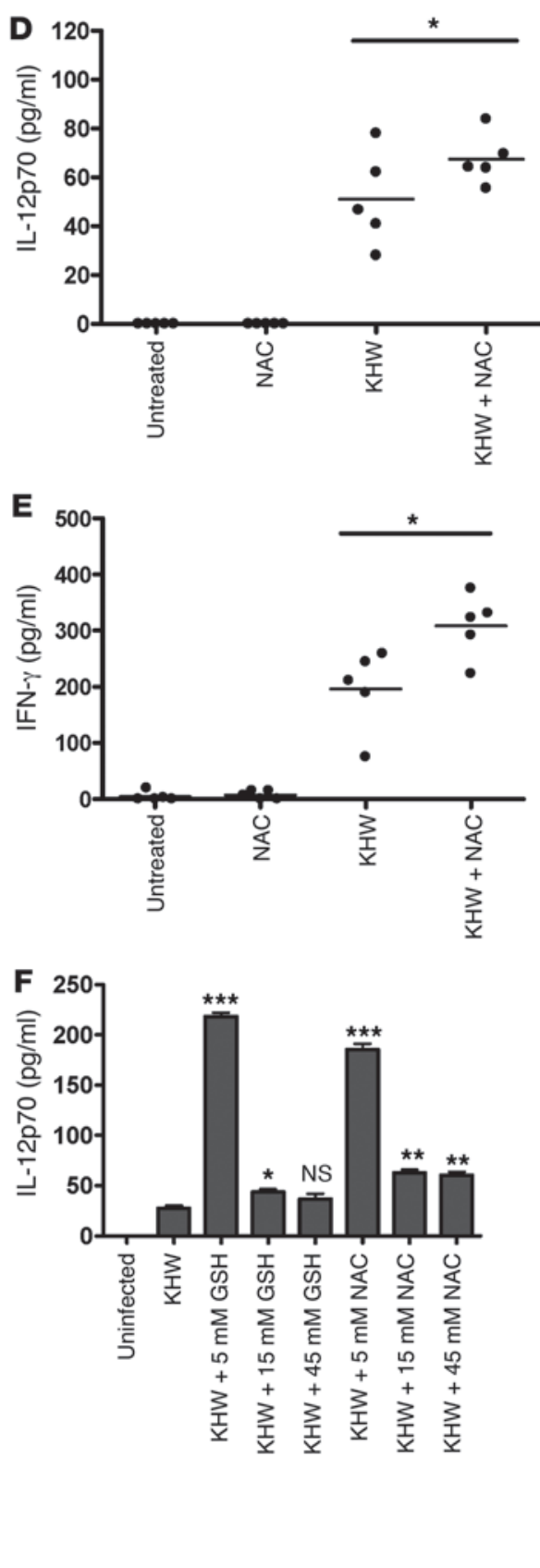

Figure 4

Effects of GSH modulators on B. pseudomallei -induced IL-12 and IFN- $\gamma$ production in PBMCs isolated from healthy donors. PBMCs were pretreated with either GSH (5 mM) or DEM $(60 \mu \mathrm{M})$ prior to infection with B. pseudomallei for 24 hours. (A) IL-12 and (B) IFN- $\gamma$ production were determined by ELISA. (C) PBMCs were left untreated or pretreated with $\mathrm{GSH}(5 \mathrm{mM})$ for 24 hours prior to infection. The production of 12 cytokines (IL- $1 \alpha$, IL-1 $\beta$, IL-2, IL-4, IL-6, IL-8, IL-10, IL-12p70, IL-17A, IFN- $\gamma$, TNF- $\alpha$, and GM-CSF) was determined by multiplex ELISA. (D and E) PBMCs were pretreated with NAC $(5 \mathrm{mM})$ for 24 hours before infection, and (D) IL-12 and (E) IFN- $\gamma$ production were determined by ELISA. (F) PBMCs were pretreated with the indicated concentrations of either GSH or NAC for 24 hours prior to infection. IL-12 production was determined by ELISA. Each symbol represents data obtained from 1 study subject. Horizontal lines for the dot plots show the mean values for that particular treatment group. Data for the bar charts are presented as mean \pm SEM. ${ }^{* * *} P<0.001 ;{ }^{* *} P<0.01$; ${ }^{\star} P<0.05$. n.s. $=P>0.05$. tion in IFN- $\gamma$ production, compounding the poor respiratory burst, thereby leading to a decrease in microbicidal killing efficacy in diabetic subjects as compared with healthy controls. The defective production of IL-12 and IFN- $\gamma$ after bacterial infection of diabetic cells was highly specific because other proinflammatory cytokines, such as TNF- $\alpha$, IL-1 $\beta$, IL-18, IL-8, and antiinflammatory cytokines, such as IL-10, were similarly produced by cells from both groups of individuals. Therefore, our work has uncovered the unique link between GSH concentrations in PBMCs and susceptibility to intracellular bacterial infections in type 2 diabetes specifically through the action of IL-12. Our model provides an explanation for the increased susceptibility of type 2 diabetic patients to melioidosis and possibly tuberculosis.
Past studies had found that disease prognosis for melioidosis patients with underlying diabetes did not seem to be worse than for those without diabetes, and there appeared to be an inverse correlation between diabetes and mortality; recently, glyburide or glybenclamide has been identified as the confounding factor in those studies (25). More than half of all diabetic melioidosis patients were prescribed this oral hypoglycemic agent, and those on glyburide had a survival advantage compared with nondiabetic melioidosis patients and with diabetic melioidosis patients on other diabetic medications, such as metformin and insulin. The beneficial effect of glyburide was attributed to its antiinflammatory nature (25), as it had been shown to be able to inhibit NLRP3 inflammasome activation (12). Our findings support the inter- 
Table 2

Correlation among glycemic control, GSH/GSSG ratio, bacterial loads, and IL-12 and IFN- $\gamma$ concentrations in PBMCs

$\begin{array}{lcccc} & \text { Healthy } & \text { Good glycemic control } & \text { Poor glycemic control } & P \text { value } \\ n & 10 & 9 & 10 & - \\ \text { HbA1c } & \text { NA } & 6.64 \pm 0.46 & 10.44 \pm 2.51 & 0.001^{\mathrm{A}} \\ \text { GSH/GSSG } & 7.68 \pm 0.84 & 8.76 \pm 1.61 & 4.46 \pm 0.75 & 0.001^{\mathrm{A}} \\ \mathrm{BMI} & 22.59 \pm 3.02 & 29.31 \pm 4.65 & 25.66 \pm 10.65 & 0.05^{\mathrm{B}} \\ \text { Bacterial loads (CFU/ml) } & 2816 \pm 1766 & 3401 \pm 3392 & 8305 \pm 4330 & 0.01^{\mathrm{C}} \\ & & & & 0.05^{\mathrm{D}} \\ \mathrm{IL}-12 \mathrm{p} 70(\mathrm{pg} / \mathrm{ml}) & 48.84 \pm 27.77 & 50.70 \pm 28.91 & 24.93 \pm 20.97 & 0.05^{\mathrm{E}} \\ \text { IFN- } \gamma(\mathrm{pg} / \mathrm{ml}) & 248.86 \pm 78.61 & 221.37 \pm 103.18 & 138.93 \pm 64.77 & 0.01^{\mathrm{C}} \\ & & & & 0.05^{\mathrm{D}}\end{array}$

Values are expressed as mean \pm SEM from 10 individuals per group except for the good glycemic control group of 9 individuals. ${ }^{A} P<0.001$, differences between poor glycemic control and either healthy or good glycemic control groups. ${ }^{\mathrm{B}} P<0.01$, differences between healthy and good glycemic control groups. ${ }^{C} P<0.01$, differences between poor glycemic control and healthy groups. ${ }^{D} P<0.05$, differences between poor glycemic control and good glycemic control groups. $\mathrm{E} P<0.05$, differences between poor glycemic control and either healthy or good glycemic control groups. NA, not applicable.

pretation that low intracellular GSH levels in diabetics are critical in allowing better initiation of the intracellular infection and provide an explanation for the increased susceptibility of diabetic individuals to melioidosis. The low intracellular GSH/GSSG ratio could also affect disease progression and outcome, provided the confounding effect of glyburide is removed. Our depletion of GSH in the mouse model of melioidosis supports the conclusion that GSH deficiency contributes to decreased IL-12 production, increased disease susceptibility, and poorer disease outcome.

Diabetes mellitus is also recognized as a major risk factor for tuberculosis, and several studies have shown that coaffliction with tuberculosis and diabetes is common in both low- and high-income countries (1). Our ex vivo data support the interpretation that GSH deficiency in PBMCs from diabetics accounts for the impaired ability to control tuberculosis infection via a defect in IL-12 production. Consistently, GSH supplementation in mice has been reported to improve control of tuberculosis infections via a direct cytolytic effect of GSH on the bacteria and by improving NK cell activity and Th1 response, resulting in better $\mathrm{T}$ cell control of infection, although the underlying mechanisms are unknown (26). Furthermore, low concentrations of NAC used to increase GSH levels augmented bacillus Calmette-Guérin-triggered IL-12 production through increased c-rel translocation into the nucleus for NF- $\mathrm{KB}$ activation (18). These data support our interpretation that susceptibility to tuberculosis in diabetic patients is contributed by the GSH/GSSG imbalance, which affects IL-12 production and subsequent bacterial control. Although this study did not examine the adaptive responses of diabetic patients with low GSH/GSSG ratio to B. pseudomallei or M. tuberculosis, it is possible the adaptive Th1 responses are also compromised.

Redox balance and oxidative stress in the diabetic condition have been examined previously, but those studies reported lower intraerythrocytic or plasma concentrations of GSH in type 2
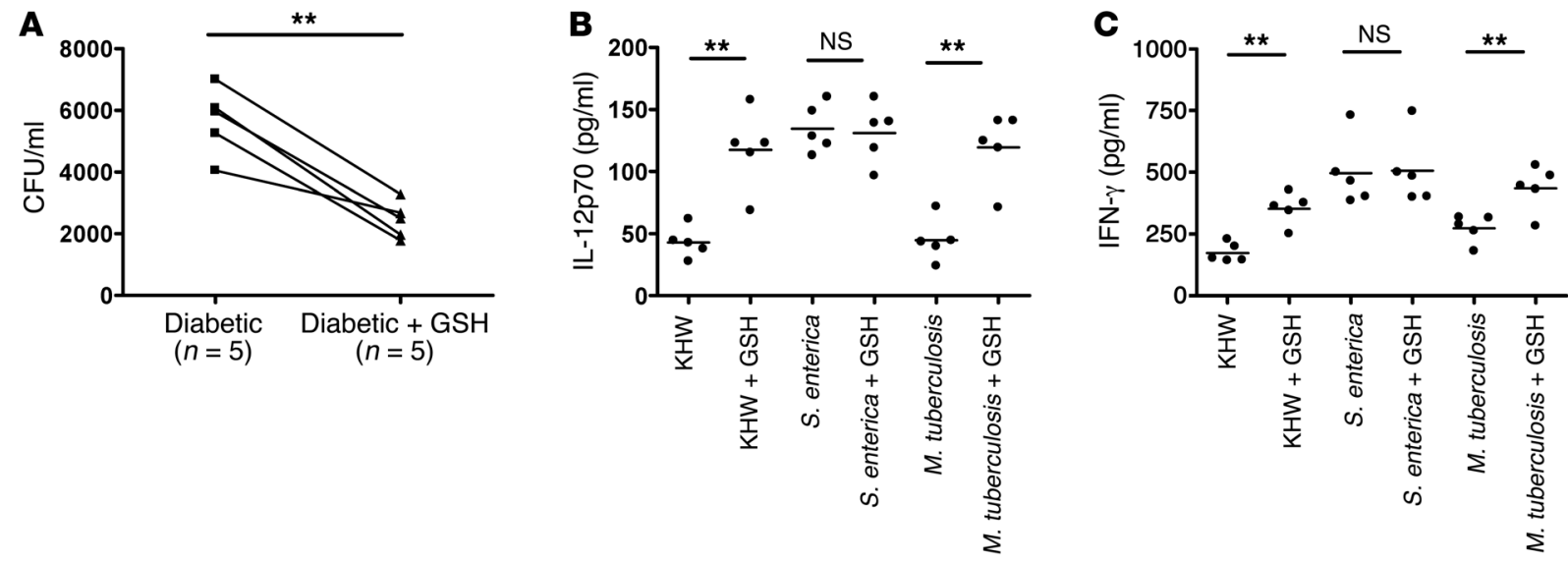

\section{Figure 5}

GSH supplementation reduces intracellular bacterial loads and improves IL-12 and IFN- $\gamma$ production in PBMCs isolated from diabetics with poor glycemic control. (A) PBMCs isolated from diabetics with poor glucose control were left untreated or pretreated with GSH (5 mM) prior to infection with B. pseudomallei, and intracellular bacterial loads were determined 8 hours after infection. (A) Each symbol denotes data obtained from 1 study subject either before or after GSH treatment. Values obtained from the same study subject before and after treatment are joined by a line. PBMCs were left untreated or pretreated with GSH $(5 \mathrm{mM})$ prior to infection with B. pseudomallei, S. enterica, or M. tuberculosis, and the amount of (B) IL-12 and (C) IFN- $\gamma$ production was determined. (B and C) Each symbol denotes data obtained from 1 study subject. Horizontal lines in the dot plots represent the mean values for that particular experimental group. ${ }^{* *} P<0.01 ;$ n.s. $=P>0.05$. 

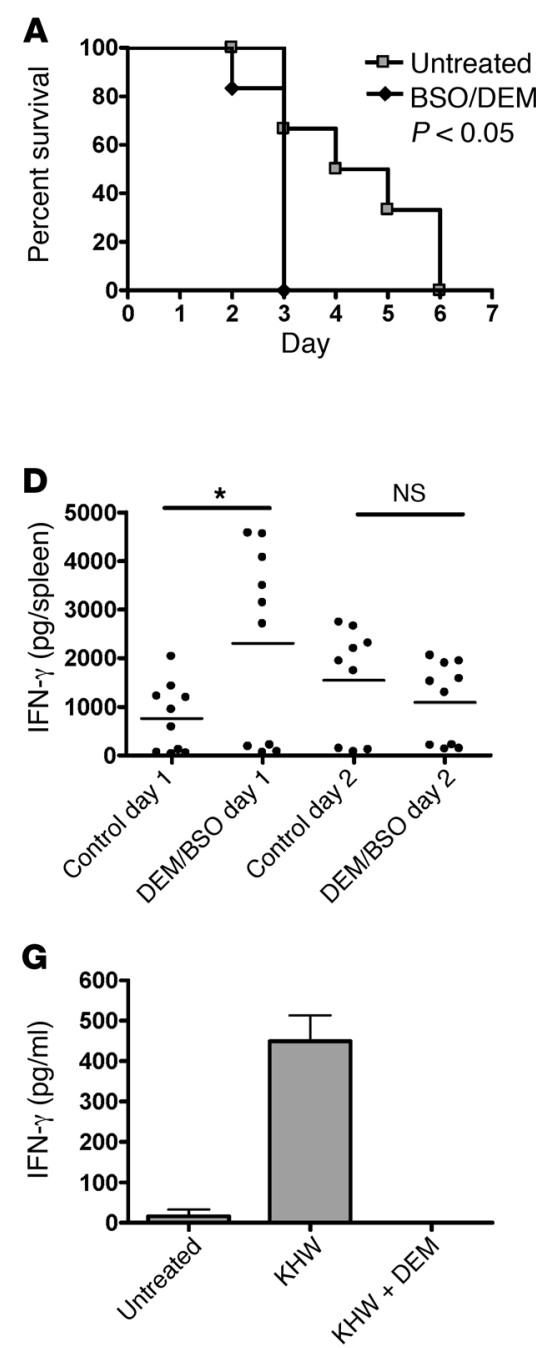

B
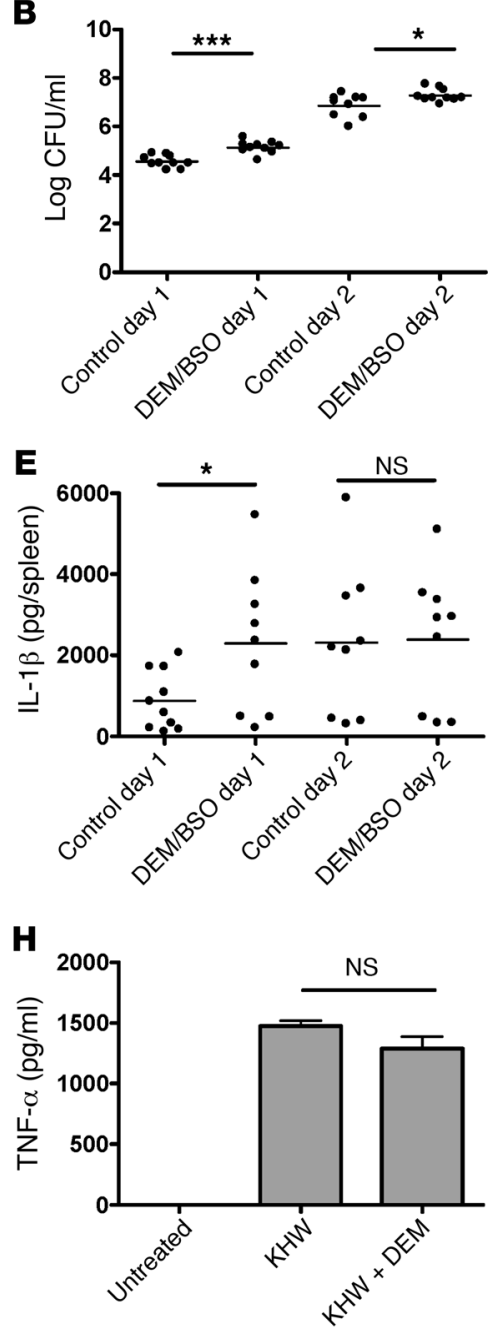

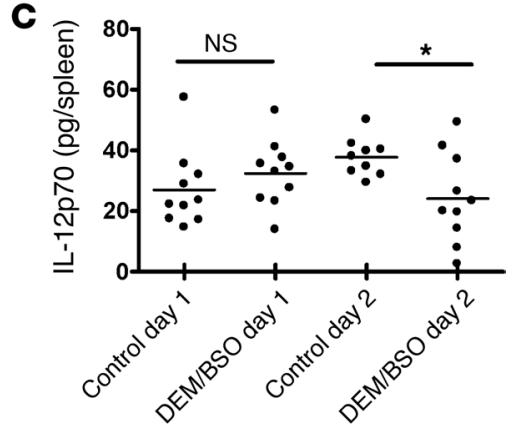

$\mathbf{F}$

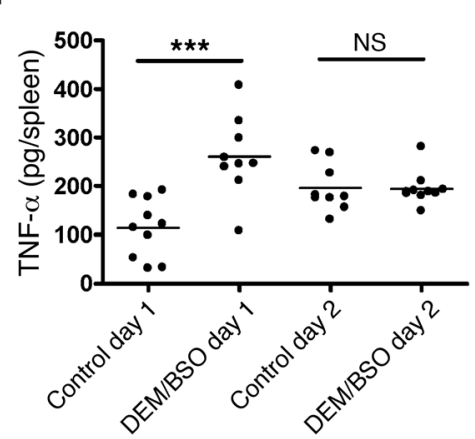

\section{Figure 6}

Effect of GSH depletion in murine model of melioidosis. BALB/c mice were left untreated or treated with DEM/BSO intraperitoneally 2 hours prior to infection with B. pseudomallei. (A) Survival of control or DEM/BSO-depleted mice following infection. Statistical significance was determined using the log-rank test. (B) Bacterial loads in the spleens of control or DEM/BSO-treated mice at day 1 and day 2 after infection. (C) IL-12p70 (D) IFN- $\gamma$, (E) IL-1 $\beta$, and (F) TNF- $\alpha$ concentrations in spleens of untreated and DEM/BSO-treated groups. Results shown in $\mathbf{B}-\mathbf{F}$ are combined from 2 independent sets of experiments. ( $\mathbf{G}$ and $\mathbf{H})$ Splenocytes were pretreated with DEM $(50 \mu \mathrm{M})$ for 2 hours before infection with $B$. pseudomallei for 36 hours. (G) IFN- $\gamma$ and (H) TNF- $\alpha$ production were determined by ELISA. Each symbol represents data obtained from 1 animal. The horizontal lines for the dot plots show the mean values for particular experimental groups. Data for the bar charts are presented as mean \pm SEM. ${ }^{* *} P<0.001 ;{ }^{*} P<0.05$; n.s. $=P>0.05$.

diabetic versus healthy individuals $(27,28)$. We found that intracellular GSH concentrations and the GSH/GSSG ratios in white blood cells of diabetic patients were also significantly lower than those from healthy controls. This compromised the ability of the innate immune cells to produce, in response to certain infections, IL-12, a key cytokine necessary for the initiation of a proinflammatory response due to its ability to activate the production of IFN- $\gamma$. IFN- $\gamma$ is important for activating phagocytes, such as monocytes, so that they become microbicidal. Several studies had shown that depletion of GSH in APCs could shift T cell maturation to a Th2 response, whereas GSH or NAC supplementation resulted in a Th1 response via the production of IL-12 from the $\operatorname{APCs}(17,18,29,30)$, although the mechanism remains unknown. In contrast, Mazzeo et al. found that NAC and GSH decreased
IL-12p70 dimer formation (31). Our results are in agreement with the majority of the studies in which we have shown that GSH concentrations in PBMCs of diabetic patients affect their acute response to certain bacterial infections through the production of IL-12 and IFN- $\gamma$. The key difference in the latter study (31) could be the incubation of GSH and NAC throughout the stimulation, which could affect IL-12p70 dimer formation directly, whereas we removed GSH/NAC prior to bacterial infection so that the effect of GSH/NAC was solely to increase the GSH/GSSG ratio. Since we have shown that decreased IL-12 response in diabetics is not a general defect in IL-12 production because TLR agonists and S. enterica could trigger comparable IL-12 production from diabetic and healthy cells, the use of a different bacterial agent such as S. aureus (31) would also result in a different outcome. 
We believe that B. psendomallei and M. tuberculosis likely trigger a signaling pathway different from $S$. enterica that is susceptible to the changes in intracellular GSH concentrations, even though IL-12 and IFN- $\gamma$ have been shown to be important for the control of all 3 intracellular pathogens $(13,32)$. The MyD88- and TRIF-dependent signaling pathways appeared not to be involved directly because stimulation by specific TLR agonists produced similar IL-12 responses from the monocytes of both diabetic and healthy donors. Instead, our data support that B. pseudomallei and $M$. tuberculosis, but not S. enterica, induce an alternative pathway that subsequently triggers IL-12 production. It has been shown that IL-15 produced from the crosstalk between conventional DCs (cDCs) and plasmacytoid DCs drove the production of IL-12 from cDCs (33). IL-15 is produced from many cell types, including monocytes (34). It is possible that IL-15 could be differentially produced between diabetic and healthy cells upon bacterial infection, as elevated plasma concentrations of IL-15 were indeed present in patients with severe melioidosis (23). Differences in signaling from other cell types could also accentuate the differential IL-12 production. It has been documented that $B$. pseudomallei infection induced early IFN- $\gamma$ production by NK cells and bystander T cells with memory phenotype both in mice and humans (35-37). These early sources of IFN- $\gamma$ represent the priming signals in our monocyte model, necessary to help monocytes produce IL-12 when triggered by bacterial infection. This scenario would be similar to the 3 -cell model involved in the priming of Th1 polarization, in which NK or $\gamma \delta$ T cells produce IFN- $\gamma$ to provide help to DCs triggered by bacterial Pathogen Associated Molecular Patterns (PAMPs), so that the DCs can provide antigen and costimulatory signals to prime naive $\mathrm{T}$ cell differentiation (38). It is thus conceivable that, besides the defective IL-12 production in monocytes due to the low GSH/ GSSG ratio, the early IFN- $\gamma$ priming signals from NK or activated/ memory $\mathrm{T}$ cells could also be suboptimal in diabetic individuals with poor glycemic control. Thus, the low GSH concentrations in NK and T cells would affect the production of the priming signals. GSH could potentially affect cell signaling in many ways, such as modulating intracellular free radicals, maintaining the thiol status of proteins, and covalently binding cysteine moieties in cytosolic proteins (S-glutathionylation) (39). Furthermore, the sensitivity of tyrosine phosphatases to intracellular thiol status has been shown to be important in regulating the immune response $(40,41)$. For example, a GSH deficiency in HIV-infected CD4 ${ }^{+} \mathrm{T}$ cells impaired tyrosine phosphatase activity, which could be restored by the presence of NAC (42). The system is no doubt a complicated one, and future studies would require the simultaneous examination of several cell types and their relative roles as well as how their signaling pathways could be affected by GSH concentrations.

Three mechanisms have been proposed to explain the reduced levels of GSH under hyperglycemic conditions in type 2 diabetes. First, glucose affects GSH synthesis by decreasing the activity of the rate-limiting enzyme $\gamma$-glutamylcysteine ligase $(39,43)$. Second, excess glucose is converted to sorbitol via the polyol pathway, consuming NADPH in the process. As NADPH is a cofactor required for GSH regeneration from GSSG, the consumption of NADPH affects the regeneration of GSH (16). Third, advanced glycation end-products (AGE) induce formation of superoxide and hydrogen peroxide, which deplete GSH $(44,45)$. We have shown that diabetics with good glycemic control (HbA1c $\leq 7)$ indeed had intracellular GSH concentrations similar to those of healthy controls. The GSH/GSSG ratio only became aberrant in patients with
HbA1c greater than or equal to 8.0. The GSH/GSSG ratio is tightly correlated with IL-12-IFN- $\gamma$ production and intracellular bacterial control. Our results thus argue for the potential use of that intracellular GSH to GSSG ratio as a reliable biomarker to predict the state of health of the immune system of diabetic patients and their ability to control certain types of intracellular bacterial infections. It remains to be seen whether our prediction model can be extended beyond B. psendomallei and M. tuberculosis to other bacteria or viruses such as influenza (46).

Despite advances in treatment for melioidosis, the case fatality ratio has remained constant at around $15 \%-20 \%$ in Australia and $40 \%-50 \%$ in Thailand (47). The finding, in 1989, that ceftazidime halves the mortality of severe melioidosis was seminal in establishing ceftazidime as the drug of choice used for the intensive phase of the therapy (48), and no other antibiotic regimens since then have been shown to have a mortality benefit (47). Eradication phase of the therapy involves the use of trimethoprim-sulfamethoxazole (TMP-SMX) with doxycycline or TMP-SMX alone (47). G-CSF was originally thought to provide significant mortality benefit in melioidosis septic patients as an adjunctive therapy when compared with a historical control group in Australia (49), but in a subsequent randomized, placebo-controlled trial in Thailand, G-CSF was not associated with a mortality benefit, although it increased survival from 18.6 hours to 33 hours (50). The authors concluded that G-CSF may "buy time" by either reversing neutrophil defects associated with risk factors for melioidosis (such as diabetes) or through an antiinflammatory effect, although the high mortality overall suggests that it may not have an impact on the ultimate outcome (50). As our in vitro supplementation of NAC results in an increase in GSH concentrations and better bacterial control in diabetic cells, supplementation with antioxidants such as NAC, which has already been licensed for other clinical uses, could potentially be considered as another form of adjunctive therapy for diabetic patients with melioidosis. Its effect would likely be broader than that of G-CSF in terms of reversing the redox imbalance in all cell types to result in better immune function, particularly that of the microbicidal function of phagocytes as shown in this study. The potential application of NAC would also extend beyond usage in septic melioidosis patients to include all with poorly controlled diabetes and could be given as an adjunctive during the intensive and eradication phases. Given the relatively low cost of NAC treatment, it may even be considered as a preventive supplement in diabetics with poor glycemic control at high risk of contracting melioidosis in the hyperendemic regions.

\section{Methods}

Study population. Type 2 diabetic patients aged between 30 and 65 years were recruited from the Endocrinology Clinic, National University Hospital, Singapore. Patients must have had type 2 diabetes for a period of 5 or more years with $\mathrm{HbA} 1 \mathrm{c}$ greater than or equal to 8.0 at the time of blood donation, except for in the experiment involving the comparison between diabetic patients with good glycemic and those with poor glycemic control. For the latter experiment, the good and poor glycemic groups consisted of individuals with $\mathrm{HbA} 1 \mathrm{c}$ less than or equal to 7 or $\mathrm{HbA} 1 \mathrm{c}$ greater than or equal to 8.5 , respectively, for a period of 6 or more months. Age- and sex-matched nondiabetic volunteers served as healthy controls. Peripheral venous blood was obtained from diabetic patients or healthy controls and collected in vacutainers containing sodium heparin (BD Biosciences). PBMCs from some patients were used simultaneously for intracellular bacterial replication and cytokine assays. 
Cell manipulation. PBMCs were isolated from the blood by density gradient centrifugation using Histopaque 1077 (Sigma-Aldrich). Following cell separation, PBMCs were recovered and washed twice with $1 \times$ PBS and resuspended in antibiotic-free RPMI 1640 (Gibco; Invitrogen) supplemented with $5 \%$ heat-inactivated FBS (Gibco; Invitrogen). Primary monocytes were isolated from PBMCs by positive selection using CD14+ magnetic beads (Miltenyi Biotech). Monocytes used for all experiments were greater than $90 \%$ pure as determined by flow cytometry. Primary monocytes were treated with $100 \mathrm{ng} / \mathrm{ml}$ human recombinant IFN- $\gamma$ (BioLegend) 2 hours prior to bacterial infection or stimulation with TLR agonists. $\mathrm{Pam}_{3} \mathrm{CSK}_{4}$ (PAM), poly(I:C), and ultra-pure LPS (UP-LPS) from E. coli K12 were obtained from Invivogen, whereas recombinant $B$. pseudomallei flagellin was prepared as described previously (51). Anti-human IL-12p40 antibody and isotype control were obtained from BioLegend. Primary monocytes were treated with MyD88 (100 $\mu \mathrm{M})$ (Imgenex) or TRIF (25 $\mu \mathrm{M})$ (Invivogen) peptide inhibitors for 6 hours prior to bacterial infection. The dosage used had been titrated using positive and negative controls to ascertain sensitivity and specificity.

Bacterial infection. B. psendomallei KHW strain, a clinical isolate obtained from a patient who died from melioidosis, was used in this study (24). S. enterica was a gift from J. Kwang (Temasek Life Sciences Laboratory, Singapore). B. psendomallei or S. enterica was cultured in Luria-Bertani (LB) broth or plated on tryptic soy agar (TSA). Mid-log phase bacteria were prepared by inoculating $250 \mu \mathrm{l}$ of overnight culture into $5 \mathrm{ml}$ of LB broth and allowed to grow for 2 hours with constant agitation. Cells were infected with $\log$-phase culture of B. pseudomallei or S. enterica at an MOI of 10:1. Infected cells were centrifuged at $500 \mathrm{~g}$ for 5 minutes to allow bacteria-cell contact. Two hours after infection, $250 \mu \mathrm{g} / \mathrm{ml}$ kanamycin was added to kill off extracellular bacteria. Cells were incubated for at $37^{\circ} \mathrm{C}$ at $5 \% \mathrm{CO}_{2}$ for 24 hours before the supernatants were collected for cytokine analysis. Frozen stocks of $M$. tuberculosis $\mathrm{H} 37 \mathrm{Rv}$ wild-type strain were prepared by resuspending a log-phase bacterial culture in Middlebrook 7H9 Liquid Medium (BD Biosciences) supplemented with $25 \%$ glycerol and stored as $1 \mathrm{ml}$ single-use aliquots at $-80^{\circ} \mathrm{C}$. Bacterial concentration was determined by plating appropriate dilutions of a representative vial of stock on Middlebrook 7H11 Agar supplemented with 10\% Middlebrook OADC Enrichment (BD Biosciences) and quantifying the number of viable colonies after incubating the plates for 16 days at $37^{\circ} \mathrm{C}$ and $5 \% \mathrm{CO}_{2}$. Cells were infected with $M$. tuberculosis $\mathrm{H} 37 \mathrm{Rv}$ at an MOI of 1:1. For each infection assay, a frozen stock of H37Rv mycobacteria was defrosted and immediately diluted at the appropriate concentration in antibiotic-free cell culture medium; $500 \mu \mathrm{l}$ of inoculum was added directly to the cells, which were incubated at $37^{\circ} \mathrm{C}$ and $5 \% \mathrm{CO}_{2}$ for 24 hours before the supernatants were collected for cytokine analysis.

ELISA. Multi-Analyte ELISA Inflammatory Cytokine Panel (SA Biosciences), inclusive of IL-1 $\alpha$, IL-1 $\beta$, IL-2, IL-4, IL-6, IL-8, IL-10, IL-12p70, IL-17A, IFN- $\gamma$, TNF- $\alpha$, and GM-CSF, was used to compare cytokine production by PBMCs isolated from healthy or diabetic individuals following ex vivo infection with B. psendomallei. Analysis of IL-18 and IL-23 production in healthy and diabetic individuals was carried out using IL-18 and IL-23 ELISA kits (Bender Medsystems). Further validation of IL-12 and IFN- $\gamma$ cytokine protein concentrations was carried out using single-analyte ELISA kits from BioLegend and Bender Medsystems, respectively.

RNA isolation and quantitative real-time PCR. Total RNA was isolated using RNeasy Mini Kit (QIAGEN). Contaminating DNA in the RNA preparation was removed using column digestion with DNase I (QIAGEN). Reverse transcription was performed using the High Capacity cDNA Synthesis Kit (Applied Biosystems). Transcripts were quantified by real-time PCR using SYBR Green (Invitrogen) in a Bio-Rad iQ5 machine. The expression of cytokine mRNAs was normalized to the relative abundance of a housekeeping gene. Fold induction was calculated using the $2^{-\Delta \Delta} \mathrm{Ct}$ method (52).
Intracellular bacterial count. PBMCs were infected with B. pseudomallei at an MOI of 50:1. Kanamycin $(250 \mu \mathrm{g} / \mathrm{ml})$ was added 2 hours after infection to kill off extracellular bacteria. Cells were infected for 8 hours, after which cells were lyzed with $0.1 \%$ Triton- $X$ and the amount of intracellular bacteria was determined by serial dilution and plating on TSA plates.

Measurement of ROS. Generation of intracellular ROS was measured using the luminol-amplified chemiluminescence method. Briefly, a $0.5-\mathrm{ml}$ mixture containing $50 \mu \mathrm{l}$ of $5000 \mathrm{U} / \mathrm{ml}$ superoxide dismutase (Sigma-Aldrich), $50 \mu \mathrm{l}$ of $200000 \mathrm{U} / \mathrm{ml}$ catalase (Sigma-Aldrich), and $50 \mu \mathrm{l}$ of $0.5 \mathrm{mM}$ luminol (Sigma-Aldrich) was freshly prepared and $50 \mu \mathrm{l}$ of the mixture added to $0.5 \times 10^{6} \mathrm{PBMCs}$ inoculated with KHW at an MOI of 50:1 in a final volume of $0.5 \mathrm{ml}$. Luminescence was measured using a single-tube luminometer (Turner Designs).

Modulation and determination of intracellular GSH and GSSG concentrations. To increase intracellular GSH, PBMCs were treated with either $5 \mathrm{mM} \mathrm{GSH}$ reduced ethyl ester (GSH-OEt) (Sigma-Aldrich) or 5 mM NAC (SigmaAldrich). Cells were treated with $60 \mu \mathrm{M}$ DEM (Sigma-Aldrich) to deplete intracellular GSH. Cells were incubated with GSH modulators in RPMI 1640 (Gibco; Invitrogen) supplemented with 1\% heat-inactivated FBS for 6 hours. Cells were washed twice with $1 \times$ PBS and resuspended in fresh antibiotic-free medium prior to bacterial infection. Intracellular GSH and GSSG concentrations in PBMCs were determined using Glutathione Colorimetric Detection Kit (Arbor Assays) according to the manufacturer's instructions. Animal infection studies. Female, 8- to 10-week-old BALB/c mice were purchased from the Laboratory Animals Centre (National University of Singapore). Infection of mice was carried out in an animal BSL3 facility with protocols approved by the National University of Singapore and Defence Science Organization National Laboratories IACUC committees. To measure survival, mice in both untreated and DEM/BSO-treated groups at 6 mice per group were matched by weight. For GSH depletion, DEM (Sigma-Aldrich) $(0.8 \mathrm{ml} / \mathrm{kg}$ ) and BSO (Sigma-Aldrich) $(700 \mathrm{mg} / \mathrm{kg})$ were administered to mice 2 hours prior to infection with a dose of 2,000 CFU by intraperitoneal injection, and animals were monitored daily for survival. To measure the bacterial loads and cytokine production over time, 2 independent experiments were performed and the data combined in the same graph. Mice in both untreated and DEM/BSO-treated groups were matched by weight. For GSH depletion, DEM $(0.8 \mathrm{ml} / \mathrm{kg})$ and BSO (700 $\mathrm{mg} / \mathrm{kg}$ ) were administered to mice 2 hours prior to infection with a dose of $1,000 \mathrm{CFU}$ by intraperitoneal injection. Infection dose was reduced to prevent premature death of animals before the time points. At 1 and 2 days after infection, 4 mice per group in the first experiment and 6 mice per group in the second experiment were sacrificed and spleens removed aseptically and homogenized in PBS. For organ load determination, the spleen homogenates were serially diluted accordingly and plated on Ashdown agar plates. For cytokine determination, the spleen homogenates were filtered through 0.2- $\mu \mathrm{m}$ syringe filters (Millipore) before ELISA was carried out with the following cytokine ELISA kits: mouse IL-1 $\beta$ (BioLegend), IL12 p70 (BioLegend), IFN- $\gamma$ (Bender Medsystems), and TNF- $\alpha$ (eBiosciences). Only 3 data points for all parameters were available for the untreated mice at day 2 after infection in the first experiment due to problems with the infection of 1 mouse that had to be discarded.

In vitro splenocyte studies. Eight- to ten-week-old female BALB/c mice were killed by carbon dioxide asphyxiation and spleens removed aseptically. Collagenase $\mathrm{D}$ (Roche) treatment and homogenization were carried out on the isolated spleen before passing it through sterile nylon mesh to generate single-cell suspensions. Splenocytes were pretreated with DEM for 2 hours before infection. Splenocytes were infected with B. psendomallei at an MOI of 5:1. Two hours after inoculation with bacteria, $250 \mu \mathrm{g} / \mathrm{ml}$ of kanamycin was added to inhibit the growth of the extracellular bacteria. For surface staining of cells, anti-CD19-APC (clone 6D5; BioLegend), anti-F480-APC 
(clone BM8; BioLegend), anti-CD3-FITC (clone 145-2C11; BD Biosciences - Pharmingen), anti-I-A/I-E-FITC (clone M5/114.15.2; BioLegend), and anti-CD11c-APC (clone N418; Miltenyi Biotec) were used. Supernatants were collected after 36 hours for IL-12p70, TNF- $\alpha$, and IFN- $\gamma$ ELISAs, whereas splenocytes were harvested from wells at 10 hours after infection for surface staining.

Statistics. Statistical significance was determined by performing paired or unpaired 2-tailed Student's $t$ test. Paired tests were performed for data in Figure 2 and Figure 5A. For survival curves, statistical significance was determined using log-rank test. Differences were considered significant at $P<0.05$.

Study approval. All human studies were approved by the National University of Singapore Institutional Review Board, and written informed consent was obtained from all volunteers prior to their participation in the study. All animal studies were approved by the National University of Singapore as well as the Defence Science Organization Institutional Animal Care and Use Committees.

\section{Acknowledgments}

This study was funded by grants NMRC/1110/2007 from the National Medical Research Council and the MINDEF/NUS/JPP/11/05 from Ministry of Defence and National University of Singapore Joint Applied R\&D Co-Operation Programme, Singapore. We thank Isabelle Chen for providing logistical support for the project.

Received for publication May 27, 2011, and accepted in revised form March 7, 2012.

Address correspondence to: Yunn-Hwen Gan, Department of Biochemistry, Yong Loo Lin School of Medicine, National University of Singapore, 8 Medical Drive, Singapore 117597. Phone: 65.6516.3678; Fax:65.6779.1453; E-mail:yunn_hwen_gan@nuhs.edu.sg.

Kai Soo Tan's present address is: Faculty of Dentistry, National University of Singapore, Singapore.
1. Dooley KE, Chaisson RE. Tuberculosis and diabetes mellitus: convergence of two epidemics. Lancet Infect Dis. 2009;9(12):737-746.

2. Ritz E, Orth SR. Nephropathy in patients with type 2 diabetes mellitus. $N$ Engl J Med. 1999; 341(15):1127-1133.

3. Renard C, Van Obberghen E. Role of diabetes in atherosclerotic pathogenesis. What have we learned from animal models? Diabetes Metab. 2006;32(1):15-29.

4. Yamagishi SI, Nakamura K, Matsui T, Takenaka K, Jinnouchi Y, Imaizumi T. Cardiovascular disease in diabetes. Mini Rev Med Chem. 2006;6(3):313-318.

5. Pop-Busui R, Sima A, Stevens M. Diabetic neuropathy and oxidative stress. Diabetes Metab Res Rev. 2006;22(4):257-273.

6. Moutschen MP, Scheen AJ, Lefebvre PJ. Impaired immune responses in diabetes mellitus: analysis of the factors and mechanisms involved. Relevance to the increased susceptibility of diabetic patients to specific infections. Diabetes Metab. 1992;18(3):187-201.

7. White NJ. Melioidosis. Lancet. 2003;361(9370): 1715-1722.

8. Cheng AC, Currie BJ. Melioidosis: epidemiology, pathophysiology, and management. Clin Microbiol Rev. 2005;18(2):383-416

9. Simpson AJH, Newton PN, Chierakul W, Chaowagul W, White NJ. Diabetes mellitus, insulin, and melioidosis in Thailand. Clin Infect Dis. 2003;36(5):e71-e72.

10. Hassan $M$, et al. Incidence, risk factors and clinical epidemiology of melioidosis: a complex socioecological emerging infectious disease in the Alor Setar region of Kedah, Malaysia. BMC Infect Dis. 2010;10:302.

11. Chanchamroen S, Kewcharoenwong C, Susaengrat W, Ato M, Lertmemongkolchai G. Human polymorphonuclear neutrophil responses to Burkholderia psendomallei in healthy and diabetic subjects. Infect Immun. 2009;77(1):456-463.

12. Zhou R, Tardivel A, Thorens B, Choi I, Tschopp J. Thioredoxin-interacting protein links oxidative stress to inflammasome activation. Nat Immunol. 2010;11(2):136-140.

13. Santanirand P, Harley VS, Dance DA, Drasar BS, Bancroft GJ. Obligatory role of gamma interferon for host survival in a murine model of infection with Burkholderia pseudomallei. Infect Immun. 1999;67(7):3593-3600.

14. Breitbach K, Klocke S, Tschernig T, van Rooijen N, Baumann U, Steinmetz I. Role of inducible nitric oxide synthase and NADPH oxidase in early control of Burkholderia pseudomallei infection in mice. Infect Immun. 2006;74(11):6300-6309.

15. Oppmann B, et al. Novel p19 protein engages IL$12 \mathrm{p} 40$ to form a cytokine, IL-23, with biological activities similar as well as distinct from IL-12.
Immunity. 2000;13(5):715-725.

16. Brownlee M. Biochemistry and molecular cell biology of diabetic complications. Nature. 2001; 414(6865):813-820.

17. Murata Y, Ohteki T, Koyasu S, Hamuro J. IFNgamma and pro-inflammatory cytokine production by antigen-presenting cells is dictated by intracellular thiol redox status regulated by oxygen tension. Eur J Immunol. 2002;32(10):2866-2873.

18. Alam K, Ghousunnissa S, Nair S, Valluri VL, Mukhopadhyay S. Glutathione-redox balance regulates c-rel-driven IL-12 production in macrophages: possible implications in antituberculosis immunotherapy. J Immunol. 2010;184(6):2918-2929.

19. Williams NL, Morris JL, Rush C, Govan BL, Ketheesan N. Impact of streptozotocin-induced diabetes on functional responses of dendritic cells and macrophages towards Burkholderia psendomallei. FEMS Immunol Med Microbiol. 2011;61(2):218-227.

20. Hodgson KA, Morris JL, Feterl ML, Govan BL, Ketheesan N. Altered macrophage function is associated with severe Burkholderia pseudomallei infection in a murine model of type 2 diabetes. Microbes Infect. 2011;13(14-15):1177-1184.

21. Drew R, Miners JO. The effects of buthionine sulphoximine (BSO) on glutathione depletion and xenobiotic biotransformation. Biochem Pharmacol. 1984;33(19):2989-2994.

22. Villa P, Saccani A, Sica A, Ghezzi P. Glutathione protects mice from lethal sepsis by limiting inflammation and potentiating host defense. J Infect Dis. 2002;185(8):1115-1120.

23. Lauw FN, et al. Elevated plasma concentrations of interferon (IFN)-gamma and the IFNgamma-inducing cytokines interleukin (IL)-18, IL-12, and IL-15 in severe melioidosis. J Infect Dis. 1999;180(6):1878-1885.

24. Liu B, Koo GC, Yap EH, Chua KL, Gan YH Model of differential susceptibility to mucosal Burkholderia pseudomallei infection. Infect Immun. 2002;70(2):504-511.

25. Koh GCKW, et al. Glyburide is anti-inflammatory and associated with reduced mortality in melioidosis. Clin Infect Dis. 2011;52(6):717-725.

26. Millman AC, Salman M, Dayaram YK, Connell ND, Venketaraman V. Natural killer cells, glutathione, cytokines, and innate immunity against Mycobacterium tuberculosis. I Interferon Cytokine Res. 2008;28(3):153-165.

27. De Mattia G, Laurenti O, Bravi C, Ghiselli A, Iuliano L, Balsano F. Effect of aldose reductase inhibition on glutathione redox status in erythrocytes of diabetic patients. Metabolism. 1994;43(8):965-968.

28. Bravi MC, et al. Insulin decreases intracellular oxidative stress in patients with type 2 diabetes mel- litus. Metabolism. 2006;55(5):691-695.

29. Peterson JD, Herzenberg LA, Vasquez K, Waltenbaugh C. Glutathione levels in antigen-presenting cells modulate Th1 versus Th2 response patterns. Proc Natl Acad Sci U S A. 1998;95(6):3071-3076.

30. Koike $Y$, et al. Glutathione redox regulates airway hyperresponsiveness and airway inflammation in mice. Am J Respir Cell Mol Biol. 2007;37(3):322-329.

31. Mazzeo D, et al. Thiol antioxidants inhibit the formation of the interleukin-12 heterodimer: a novel mechanism for the inhibition of IL-12 production. Cytokine. 2002;17(6):285-293.

32. van de Vosse E, OttenhoffTHM. Human host genetic factors in mycobacterial and Salmonella infection: lessons from single gene disorders in IL-12/IL-23dependent signaling that affect innate and adaptive immunity. Microbes Infect. 2006;8(4):1167-1173.

33. Kuwajima S, Sato T, Ishida K, Tada H, Tezuka $\mathrm{H}$, Ohteki T. Interleukin 15-dependent crosstalk between conventional and plasmacytoid dendritic cells is essential for CpG-induced immune activation. Nat Immunol. 2006;7(7):740-746.

34. Carroll HP, Paunovic V, Gadina M. Signalling, inflammation and arthritis: Crossed signals: the role of interleukin-15 and -18 in autoimmunity. Rheumatology. 2008;47(9):1269-1277.

35. Haque A, et al. Role of T cells in innate and adaptive immunity against murine Burkholderia pseudomallei infection. J Infect Dis. 2006;193(3):370-379.

36. Lertmemongkolchai G, Cai G, Hunter CA, Bancroft GJ. Bystander activation of CD8+ T cells contributes to the rapid production of IFN-gamma in response to bacterial pathogens. J Immunol. 2001;166(2):1097-1105.

37. Tippayawat $\mathrm{P}$, et al. Phenotypic and functional characterization of human memory T cell responses to Burkholderia psendomallei. PLoS Negl Trop Dis. 2009;3(4):e407.

38. Corthay A. A three-cell model for activation of naïve T helper cells. Scand J Immunol. 2006;64(2):93-96.

39. Ballatori N, Krance SM, Notenboom S, Shi S, Tieu K, Hammond CL. Glutathione dysregulation and the etiology and progression of human diseases. Biol Chem. 2009;390(3):191-214.

40. Zipser Y, Piade A, Kosower NS. Erythrocyte thiol status regulates band 3 phosphotyrosine level via oxidation/reduction of band 3-associated phosphotyrosine phosphatase. FEBS Lett. 1997; 406(1-2):126-130.

41. Mustelin T, Vang T, Bottini N. Protein tyrosine phosphatases and the immune response. Nat Rev Immunol. 2005;5(1):43-57.

42. Cayota A, Vuillier F, Gonzalez G, Dighiero G. CD4+ lymphocytes from HIV-infected patients display impaired CD45-associated tyrosine phosphatase 
activity which is enhanced by anti-oxidants. Clin Exp Immunol. 1996;104(1):11-17.

43. Urata $Y$, et al. Long exposure to high glucose concentration impairs the responsive expression of gammaglutamylcysteine synthetase by interleukin-1beta and tumor necrosis factor-alpha in mouse endothelial cells. J Biol Chem. 1996;271(25):15146-15152.

44. Loske C, et al. Cytotoxicity of advanced glycation endproducts is mediated by oxidative stress. J Neural Transm. 1998;105(8-9):1005-1015.

45. Deuther-Conrad W, Loske C, Schinzel R, Dringen R, Riederer P, Münch G. Advanced glycation endproducts change glutathione redox status in SH-SY5Y human neuroblastoma cells by a hydro- gen peroxide dependent mechanism. Neurosci Lett. 2001;312(1):29-32.

46. Nencioni L, et al. Influenza A virus replication is dependent on an antioxidant pathway that involves GSH and Bcl-2. FASEB J. 2003;17(6):758-760.

47. Cheng AC. Melioidosis: advances in diagnosis and treatment. Curr Opin Infect Dis. 2010;23(6):554-559.

48. White NJ, Dance DA, Chaowagul W, Wattanagoon Y, Wuthiekanun V, Pitakwatchara N. Halving of mortality of severe melioidosis by ceftazidime. Lancet. 1989;2(8665):697-701.

49. Cheng AC, Stephens DP, Anstey NM, Currie BJ. Adjunctive granulocyte colony-stimulating factor for treatment of septic shock due to melioidosis.
Clin Infect Dis. 2004;38(1):32-37.

50. Cheng AC, et al. A randomized controlled trial of granulocyte colony-stimulating factor for the treatment of severe sepsis due to melioidosis in Thailand. Clin Infect Dis. 2007;45(3):308-314.

51. Hii CS, Sun GW, Goh JWK, Lu J, Stevens MP, Gan YH. Interleukin-8 induction by Burkholderia pseudomallei can occur without Toll-like receptor signaling but requires a functional type III secretion system. J Infect Dis. 2008;197(11):1537-1547.

52. Livak KJ, Schmittgen TD. Analysis of relative gene expression data using real-time quantitative PCR and the 2(-Delta Delta C(T)) Method. Methods. 2001;25(4):402-408. 